\title{
La factura deportiva. Diseño e implantación de un modelo de cálculo de costes para los servicios deportivos municipales"
}

\author{
JORGE GARCÍA-UNANUE \\ Departamento de Actividad Física y Ciencias del Deporte,, UNIVERSIDAD DE CASTILLA- \\ LAMANCHA,ESPAÑA.E-mail: becario.jgarcia@uclm.es \\ LEONOR GALLARDO \\ Departamento de Actividad Física y Ciencias del Deporte, UNIVERSIDAD DE CASTILLA- \\ LA MANCHA, ESPAÑA. E-mail: Leonor.Gallardo@uclm.es
}

JOSÉ LUIS FELIPE

Departamento de Teoría, Recreación y Organización del Deporte, UNIVERSIDAD EUROPEA DE MADRID, ESPAÑA. E-mail: Joseluis.Felipe@uem.es

\begin{abstract}
RESUMEN
Las entidades municipales, incluidos sus servicios deportivos, están inmersas en un necesario proceso de modernización. El objetivo de este trabajo es diseñar un modelo de cálculo de costes específico para los servicios deportivos municipales, para facilitar dicho proceso. Para ello se optó por una metodología participativa, diseñando el modelo e implantándolo en dos casos reales, en colaboración con ambas entidades. El modelo propuesto se ha podido implantar correctamente y se ha adaptado tanto a sus características particulares como a sus necesidades. Los gestores de los servicios deportivos destacan de él tanto la utilidad de los resultados tanto de forma inmediata, como en la planificación a medio y largo plazo.
\end{abstract}

Palabras clave: Servicios deportivos municipales, cálculo de costes, modernización, instalaciones deportivas.

\section{The Sport Bill. Design and Implementation of a Costing Model for Municipal Sports Services}

\begin{abstract}
The Municipal entities, including its sports services, are engaged in a necessary process of modernization. The objective of this work is to design a costing model specific for the municipal sports services, to facilitate this process. For this purpose, we opted for an interventionist approach, designing and implanting the model in two case studies, working with both entities. The proposed model has been implemented correctly and has adapted to entities characteristics and needs. The managers of sports services highlight the usefulness of model results, immediately and in medium and long term planning.
\end{abstract}

Keywords: Municipal Sports Services, Costing, Modernization, Sport Facilities.

Clasificación JEL: H70, L83, M40

\footnotetext{
* En este artículo se presentan aportaciones obtenidas por los autores como consecuencia de la participación en un proyecto de investigación subvencionado por el Consejo Superior de Deportes (039/UPB10/12).
}

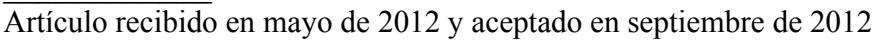

Artículo disponible en versión electrónica en la página www.revista-eea.net, ref. ə-30219 


\section{INTRODUCCIÓN}

En la actualidad el sector público está inmerso en un proceso de modernización, denominado de forma general la Nueva Gestión Pública (New Public Management, en adelante NPM), basado en una serie de reformas hacía la mejora del rendimiento en estas organizaciones (Hood 1991, 1995; Lapsley y Pallot, 2000). Este nuevo enfoque en la gestión está basado en la búsqueda de un mejor resultado, la evaluación del desempeño y una mayor competitividad (Barretta y Busco, 2011; Helmuth, 2010), adquiriendo una orientación de mercado y ofreciendo el mejor servicio al consumidor o cliente (Aberbach y Christensen, 2005; Helmuth, 2010; Walker et al., 2011). Esta nueva ideología surge de la necesidad de aumentar la eficiencia y la eficacia en la gestión de los servicios públicos, incluyendo y adaptando herramientas y métodos del sector privado a la administración pública (Budding, 2004; Van Helden et al., 2010).

La administración local ha estado especialmente afectada por estas reformas (Buch Gómez y Cabaleiro Casal, 2011; Kluvers y Tippett, 2011). Un aumento de la presión respecto a su gestión económica y un aumento de la demanda de calidad por parte de la población (Carmo y Padovani, 2012), ha desembocando en un proceso de adaptación y cambio en las entidades municipales, siendo el ente que mayor influencia directa tiene con los ciudadanos, a través de la prestación de sus servicios. Uno de los aspectos más relevantes que afecta a las entidades locales y a sus diferentes áreas es el proceso de descentralización (Balaguer-Coll et al., 2010), lo que provoca que cada área tenga más autonomía en la toma de decisiones y por lo tanto, la oportunidad de llevar un mayor control sobre sus acciones en busca del mejor servicio y el mejor resultado (Boyne et al., 2004).

Desde esta perspectiva, la gestión del rendimiento y la evaluación de resultados han sido el principal cambio que se ha dado en el sector público (Jackson y Lapsley, 2003), y por lo tanto, el principal foco de investigación en la administración local y en la prestación de servicios municipales, atendiendo a las serias dificultades que entraña la medición del logro en dichas entidades (Yetano, 2009). Encontramos investigaciones que se han centrado en métodos noparamétricos como el Análisis Envolvente de Datos (Afonso y Fernandes, 2006; Giménez y Prior, 2007; Worthington, 2000; Worthington y Dollery, 2002). Por otro lado encontramos la investigación que ha fijado como objetivo la identificación de indicadores de rendimiento estandarizados, en busca del benchmarking, y su aplicación en diferentes servicios municipales a través de diferentes métodos como el cuadro de mando integral (Padovani et al., 2010; Pollanen et al., 2011; Rodríguez Bolivar et al., 2010; Yetano, 2009). Entre los problemas encontrados a la hora de implantar dichos indicadores destacan obstáculos por las características que presentan este tipo de organizaciones, como la excesiva centralización en el control de la información así como canales de información 
inadecuados, el factor humano, debido al escepticismo sobre su utilidad o simplemente la falta de motivación y además obstáculos relacionados con la cultura de costes, debido a la inexistencia de modelos de cálculo o los problemas en la definición y registro de la información sobre los recursos consumidos (Navarro Galera et al., 2008; Ortiz Rodríguez et al., 2006).

Entre todos estos avances destacamos la utilización de la contabilidad de gestión, a través del cálculo de costes, ya que proporciona información de gran utilidad para la toma de decisiones, facilita el control de la organización y por lo tanto la evaluación y el establecimiento de indicadores.

\subsection{La contabilidad de gestión en el sector público y la administración local}

La contabilidad de gestión se define como una práctica que facilita la planificación, la evaluación y el control de la entidad por parte de sus responsables (Malmi y Granlund, 2009). Ésta se basa en la incorporación de métodos que permitan obtener información relevante para la toma de decisiones, la cual no puede ser conseguida mediante la contabilidad general.

La contabilidad de costes es posiblemente la forma más extendida de implantar la contabilidad de gestión (Bjornenak, 2000). Su identificación tal y como se conoce actualmente se vincula a la revolución industrial (Caldera et al., 2007), como solución para un análisis contable en profundidad del proceso productivo de las empresas. En el caso del sector público, y en especial en las entidades municipales, nos encontramos ante una organización que opera de forma semejante a una empresa de servicios, con el añadido de la complejidad inherente de la administración municipal, en la que existe una gran interrelación y participación de diversos agentes (Barreta y Busco, 2011). Esta temática ha sido abordada por varias organizaciones, en busca de una metodología que pueda ser de utilidad para la administración pública (IGAE, 1994; 2004) y para las entidades municipales (Carrasco Díaz, 2011; FEMP, 2006) ${ }^{1}$.

\subsection{El proceso de cambio en los servicios deportivos municipales españoles}

La infraestructura deportiva ha sufrido un gran crecimiento en las últimas décadas y sobre todo, en los últimos años. El deporte es considerado hoy día

\footnotetext{
${ }^{1}$ La Intervención General de la Administración del Estado, gracias a la publicación de los Principios Generales sobre Contabilidad Analítica en las Administraciones Públicas (IGAE, 2004) sienta unas bases metodológicas, y agiliza la iniciativa de controlar los costes en todo el sector público. La Federación Española de Municipios y Provincias, por su parte, continúa el trabajo, y publica el Manual de Procedimiento para la Implantación de un Sistema de Costes en la Administración Local (Carrasco Díaz, 2011), basado en el anterior, y que sienta unas bases metodológicas adaptadas a las características de las entidades locales.
} 
como un impulsador de la economía adquiriendo una gran importancia en el crecimiento económico a escala local (Davies, 2002).

El número de instalaciones deportivas en España creció un 61,62\% de 1985 a 2005 (Gallardo et al., 2009) y el porcentaje de población que realiza actividad física y deporte se sitúa en un $45 \%$ según la última encuesta sobre los hábitos deportivos (García Ferrando y Llopis Goig, 2011). Este auge ha estado potenciado por la participación de los gobiernos municipales en la promoción y organización de actividades deportivas, adquiriendo además un gran peso en la red de infraestructuras deportivas, ya que el $80,45 \%$ de las instalaciones deportivas públicas están en posesión de los ayuntamientos según el último Censo Nacional de Instalaciones Deportivas (Gallardo, 2007). Además el 51\% de la población que hace deporte lo práctica en una instalación pública, y el $58 \%$ ha utilizado o utiliza los servicios y actividades deportivas ofertadas por el Servicio Deportivo Municipal (en adelante, SDM) de su localidad (García Ferrando y Llopis Goig, 2011).

Los SDM tienen por tanto una gran responsabilidad respecto a la promoción de la actividad física, y la obligación de dar posibilidad de acceso a toda la población a la práctica deportiva, explotando y optimizando al máximo los recursos de los que disponen (De Liu et al., 2009). Para cumplir dicho objetivo los SDM deben asegurarse de poseer unos recursos y obtener unos ingresos mínimos para poder dar sustento a dichos servicios con la mayor calidad posible.

El cálculo de costes se presenta por tanto como una herramienta de gran utilidad en los SDM, ya que debido a sus características son un área potencial para atender a las reformas del NPM, y por lo tanto, requieren de la mayor cantidad de herramientas y métodos que faciliten el control y la mejora de su gestión, haciendo frente a las dificultades de la situación actual en la búsqueda de una mayor eficacia y eficiencia para asegurar su supervivencia.

Una vez justificada la importancia de desarrollar este tipo de iniciativas y utilizar el cálculo de costes en la gestión de las entidades municipales, así como en los SDM de forma particular, esta investigación tiene como objetivo describir el proceso de diseño de un modelo de cálculo de costes específico, que atienda a la problemática de los SDM, así como su implantación en el contexto real, destacando las dificultades encontradas y las soluciones aportadas, de manera que mediante esta investigación facilite el desarrollo este tipo de estudios en organizaciones similares.

\section{DISEÑO DE LA INVESTIGACIÓN Y METODOLOGÍA}

Esta investigación se ha llevado a cabo mediante una metodología participativa en la que el investigador se introduce en la organización como un consultor-investigador, a través de un estudio de caso. Esta metodología ha sido utilizada en las investigaciones similares en entidades locales (Ter Bogt y Van 
Helden 2011) e incluso se ha llevado a cabo en SDM (Navarro Galera et al., 2008; Rodríguez Bolívar et al., 2010).

Este tipo de metodología se basa en un proceso en el que el investigador y los miembros de la organización trabajan en conjunto, para abordar y solucionar de forma progresiva un problema planteado (Ter Bogt y Van Helden, 2011). Esto implica que el investigador adquiere una posición interna en la organización a estudiar, ofreciendo un razonamiento práctico y una evidencia empírica, mientras que mantiene el vínculo con la teoría en la interpretación de los resultados (Jönson y Lukka, 2007).

Consideramos que esta metodología favorece un verdadero vínculo con la investigación y la práctica, poniendo de manifiesto la utilidad del objeto de estudio. La orientación actual tiende a utilizar esta metodología, dado los buenos resultados que produce. El trabajo empírico se consigue mediante la cooperación con la organización (Ayuso Moya y Ripoll Feliu, 2005), de manera que los datos relevantes del proceso y las estrategias aportadas puedan ser de utilidad en organizaciones o investigaciones similares.

Uno de los aspectos más criticados en la literatura de gestión, y específicamente en la contabilidad de gestión, es el vacio que existe entre los resultados obtenidos y su aplicación en el contexto real, lo que provoca que a pesar de existir investigaciones con una alta calidad metodológica no son verdaderamente relevantes en el contexto donde actúan, ya que no aportan una verdadera solución práctica a los problemas actuales que sufren dichas organizaciones, siendo el aspecto más demandado por sus responsables (Van Helden y Northcott, 2010).

La participación del investigador en la realización de este tipo de estudios permite orientar el desarrollo de iniciativas hacia la mejora de la gestión, adquiriendo una orientación práctica en el contexto que se realiza, pero que a su vez, realiza aportaciones al conocimiento académico debido a su vinculación con la teoría, incentivando a la realización de nuevas investigaciones que puedan ser trasladadas a la práctica, beneficiando a todos los agentes (Van Helden et al., 2010).

\subsection{Muestra}

La investigación se llevará a cabo a través de la implantación del modelo desarrollado en 2 municipios españoles, en sus respectivos servicios deportivos, manteniendo el anonimato de dichas organizaciones. Los municipios cuentan con una población de 10.000 y 50.000 habitantes aproximadamente y ambos SDM son gestionados de forma directa. Mediante la descripción y la puesta en común de los dos estudios realizados se ejemplificará la aplicación práctica del modelo, así como la definición y solución de las dificultades encontradas, de- 
mostrando de esta manera la viabilidad del modelo propuesto y su adaptación a la problemática específica que afecta a este tipo de organizaciones.

\subsection{Recopilación de datos}

Para la obtención de los datos se utilizó todos los métodos comunes en los estudios de caso; la entrevista, el análisis de documentos y archivos y la observación, en este caso participativa (Ayuso Moya y Ripoll Feliu, 2005; Jönsson y Lukka, 2007). Debido a la dirección y la coordinación por parte del investigador de todo el proceso, y el trabajo en cooperación con la organización, se utilizó sobre todo a la observación participativa, dada la posibilidad de recopilar datos en tiempo real (Jönsson and Lukka, 2007). Para el registro de todos los datos y la información relevante se utilizó un diario para cada uno de los estudios.

\subsection{Procedimiento}

El proceso de realización de esta investigación se dividió en 4 etapas (Tabla 1). En la primera etapa se estableció contacto con los SDM mediante reuniones personales, entre el investigador y el equipo directivo. En ambos casos se realizó una reunión de aproximadamente 60 minutos, así como varios contactos telefónicos posteriores. El objetivo de esta etapa era explicar la visión del investigador sobre la situación que atraviesa este tipo de entidades, y la propuesta de participar en la iniciativa de implantar un nuevo modelo de cálculo de costes, junto con la puesta en común de los beneficios y utilidad que se le podrían dar a los resultados del estudio.

En la segunda etapa se paso a la realización del borrador del modelo de cálculo, a través de un análisis de los métodos y de los trabajos realizados hasta el momento, abstrayendo un modelo específico para los SDM, intentando aportarle la mayor facilidad de implantación en este tipo de entidades. A este modelo se le denominó la Factura en los Servicios Deportivos Municipales, representando el coste que asumen por la prestación de cada uno de sus servicios, en este caso las actividades deportivas.

Una vez terminado el borrador del modelo se comenzó a trabajar en su implantación, acto representado por la etapa 3. Para ello el investigador trabajo en coordinación con los trabajadores de los SDM. Para facilitar el proceso se realizaron diversos encuentros formativos con los trabajadores, en los que se informo sobre los objetivos y características del modelo propuesto, así como las características básicas de la información a suministrar.

Tras ello se realizaron todos los cálculos necesarios para llegar a obtener los resultados esperados, a través de la metodología inicial, junto con las diversas modificaciones y adaptaciones que se llevaron a cabo para hacer viable el modelo propuesto en ambos casos. Tras esto se pasó a la realización de informes donde aparecían de forma estructurada los resultados obtenidos, atendiendo a 
todos los niveles que demandaban los gestores de los servicios al inicio de la investigación.

Por último se presentó la información a los gestores de los servicios, comenzando la etapa 4. Un tiempo después se organizó una última reunión, donde se evaluó de forma conjunta los resultados obtenidos y las implicaciones prácticas reales que se podían deducir de los mismos, así como sus intenciones. Además se realizó una valoración personal de la investigación en conjunto, de su relevancia y alcance, y de las mejoras que puede aportar a la gestión de los SDM.

\section{Tabla 1}

Etapas de la investigación

\begin{tabular}{|l|l|}
\hline \multicolumn{1}{|c|}{ ETAPA } & \multicolumn{1}{|c|}{ FINALIDAD } \\
\hline Etapa 1. Contacto inicial & $\begin{array}{l}\text { Contacto con los gestores de los SDM, planificación del } \\
\text { estudio y valoración de la iniciativa }\end{array}$ \\
\hline Etapa 2. Diseño del modelo de cálculo & $\begin{array}{l}\text { Desarrollo del borrador del modelo de cálculo para los } \\
\text { SDM }\end{array}$ \\
\hline $\begin{array}{l}\text { Etapa 3. Realización del estudio de costes en los } \\
\text { SDM }\end{array}$ & $\begin{array}{l}\text { Implantación del modelo de cálculo en cada uno de los } \\
\text { SDM en colaboración con su personal y realización de } \\
\text { informes }\end{array}$ \\
\hline Etapa 4. Valoración del estudio & $\begin{array}{l}\text { Valoración de la utilidad de los resultados presentados } \\
\text { en los informes, del desarrollo del estudio y del modelo } \\
\text { de cálculo propuesto }\end{array}$ \\
\hline
\end{tabular}

Fuente: Elaboración propia.

\section{PRESENTACIÓN DE LOS CASOS}

En este punto se presentará una síntesis del desarrollo del proyecto y de los estudios realizados en ambos SDM. Se destacarán las aportaciones y resultados obtenidos en las diferentes etapas del proyecto, así como las estrategias utilizadas para abordar los problemas encontrados.

\subsection{Etapa 1. Contacto inicial}

A lo largo de esta etapa se registró información de vital importancia para comprender el contexto en el que sitúa las investigaciones y de las condiciones de partida para el desarrollo del modelo. Entre las características más comentadas destaca la necesidad de satisfacer los intereses de políticos, de los trabajadores de su organización y por su puesto de los usuarios del servicio. Los responsables de los SDM intentan realizar la gestión que más convendría a la situación de sus respectivos SDM, intentando mantener el equilibrio entre todas las partes interesadas, ya que algunas actuaciones pueden influir positivamente en un sector y negativamente en otro.

Se debatió acerca de los resultados finales del estudio propuestos por los investigadores, y sobre las que esperaban encontrar los gestores de los SDM con 
los resultados finales planteados (coste por hora de cada instalación deportiva y el coste de cada actividad deportiva ofertada así como el coste unitario y por tanto su cobertura). El principal uso planteado por el investigador fue la fijación de objetivos así como la evaluación y control de todas sus instalaciones y actividades a través de la planificación, propuesta respaldada por los gestores en una visión a medio-largo plazo.

Los gestores destacan además, como uso inmediato, la utilización de la información obtenida para justificar sus programas e iniciativas ante los órganos de decisión políticos y aumentar la posibilidad de poder llevarlas a cabo finalmente, desde su completa seguridad de que mejorarán el servicio en todas sus dimensiones. Ante esta sugerencia, el equipo investigador propone otra utilidad relacionada, como es utilizar la información de cara al usuario, en campañas que muestren los costes que asumen los SDM para poder ofertar su servicio, y las ventajas que tienen los usuarios al elegirlos. De esta manera se muestra el verdadero valor que tienen inicialmente los servicios que ofertan, ya que tanto el equipo investigador como los gestores de los SDM piensan que un desconocimiento sobre el coste de producción de las actividades hace difícil la representación de su valor.

Como principal impedimento, se destaca que para la realización de este tipo de estudio se va a depender de trabajadores y de fuentes de información externas al SDM, dado que muchos de los datos que se van a necesitar son gestionados por otras áreas generales a todo el ayuntamiento debido a la excesiva centralización informativa. La labor del investigador en este caso consistió en definir claramente la información necesaria, para que el gestor pudiera demandarla a las áreas competentes, de manera que extrajeran aquella que se vinculaba únicamente al SDM.

\subsection{Etapa 2. Diseño del modelo de cálculo}

Para el desarrollo del borrador del modelo de cálculo nos hemos fundamentado principalmente en los Principios Generales sobre Contabilidad Analítica en las Administraciones Públicas (IGAE, 2004), de manera que nos aseguramos una normalización en la metodología básica, así como en la terminología y procesos principales. De igual manera, se han tenido en cuenta las sugerencias y consideraciones realizadas por los gestores.

Ajustándonos a una de las metodologías básicas en la contabilidad de costes, el modelo planteado se basa principalmente en una metodología de costes completos por centros de actividad. A través de este modelo se han relacionado la terminología propia del proceso de formación del coste a la terminología con la que operan los SDM diariamente, facilitando así su adaptación. Puesto que es la primera vez que se implanta este tipo de iniciativa, y la utilidad más valorada es 
conocer y cuantificar resultados reales, originados por la gestión que han realizado, el modelo se basará en costes reales o históricos.

Los resultados informativos principales que se pretenden conseguir con este modelo son el coste por hora de funcionamiento de las instalaciones deportivas del servicio, así como el coste total y el coste unitario de las actividades deportivas ofertadas en dichas instalaciones por el uso real que hacen de los recursos de la entidad.

Tras esta descripción encontramos dos puntos fundamentales a tener en cuenta en la propuesta de modelo, por un lado la importancia que adquieren individualmente las instalaciones deportivas, convirtiéndose en un portador de coste por sí mismas, y por otro, la determinación y el análisis individual de cada actividad deportiva ofertada por el servicio, teniendo en cuenta la instalación deportiva donde se realiza.

Las diferentes estructuras que serán objeto de cálculo en el modelo se definen como:

- Elementos de coste: será la valoración económica de todos aquellos factores productivos utilizados por específicamente por el SDM para la realización de sus labores y funciones.

- Centros de coste: corresponden a los lugares físicos o no en el que se agruparan una serie de costes como consecuencia del proceso productivo en el desarrollo de actividades deportivas. Los principales centros de costes se corresponderán con las instalaciones deportivas.

- Actividades finalistas: serán las actividades deportivas que el SDM oferta a los usuarios, ya que representan la prestación efectiva del servicio deportivo, y cuyo beneficiario directo es el usuario. Éstas pueden ser, por ejemplo, un curso para adultos, un alquiler de una instalación o una escuela deportiva.

A través de las relaciones entre las diferentes estructuras, y por lo tanto de los correspondientes repartos, se definirá el proceso a seguir para llegar al máximo nivel de desagregación del coste (Figura 1). Este proceso se estandarizará mediante el establecimiento de 3 fases, que abarcaran todas las operaciones y actuaciones necesarias para llegar a los resultados esperados.

Fase 1 o fase de identificación. En ella se realizará una clasificación de todas las instalaciones deportivas que gestiona el SDM y que representarán los centros de coste principales, así como del resto centros que participen en el proceso productivo de actividades deportivas. Se clasificaran en los siguientes grupos: 
Figura 1

Proceso de formación del coste

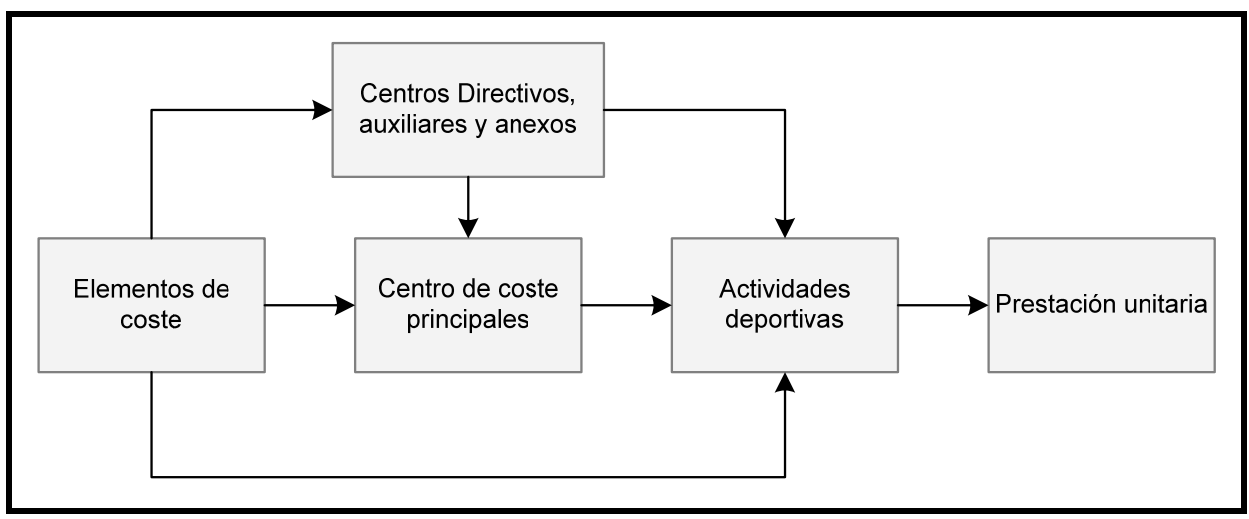

Fuente: Elaboración propia.

- Centro de coste principal: con el objetivo de facilitar el tratamiento y la agregación de los costes, éste será asumido por las instalaciones deportivas que compongan el SDM. Dada su organización, en ocasiones no se pueden identificar costes diferenciados entre instalaciones, pero sí delimitados a un grupo de las mismas, normalmente por situarse en un mismo complejo deportivo con una estructura muy cerrada. Por ello se incluye este nuevo concepto de complejo deportivo como centro de coste intermediario, en el que se agrupan unos elementos de coste que posteriormente serán asignados a las instalaciones que lo forman. De igual manera, debido a la complejidad de ciertas instalaciones deportivas, podemos encontrar varios espacios deportivos que requieran de un tratamiento individualizado, ya que sus características y utilización difieren significativamente, además de conocerse elementos de coste claramente identificados a cada uno de ellos de forma individual. Esta nueva estructura emergente recibirá el nombre de unidad deportiva, y será tratada de forma independiente en la obtención de resultados, agrupando sus costes directos y los costes de las instalaciones donde se sitúan. La unidad deportiva adquirirá por tanto la consideración de centro finalista dentro de los centros de coste principales (Figura 2).

- Centro de coste directivo y general: en este tipo de centro se agruparán los costes generales y comunes a todo el SDM.

- Centro de coste auxiliares: se identifica por los espacios auxiliares a la práctica deportiva, que participan en el proceso productivo del SDM y que afectan a instalaciones, unidades o actividades deportivas. 
- Centro de costes anexos: centro que realiza actividades no imprescindibles para el proceso productivo del SDM.

Cabe destacar que estos dos últimos centros propuestos dependerán completamente del tratamiento que se les quiera dar desde la gestión del SDM, ya que su objetivo principal es facilitar la identificación y reparto de costes.

Figura 2

Tratamiento de los centros de coste principales

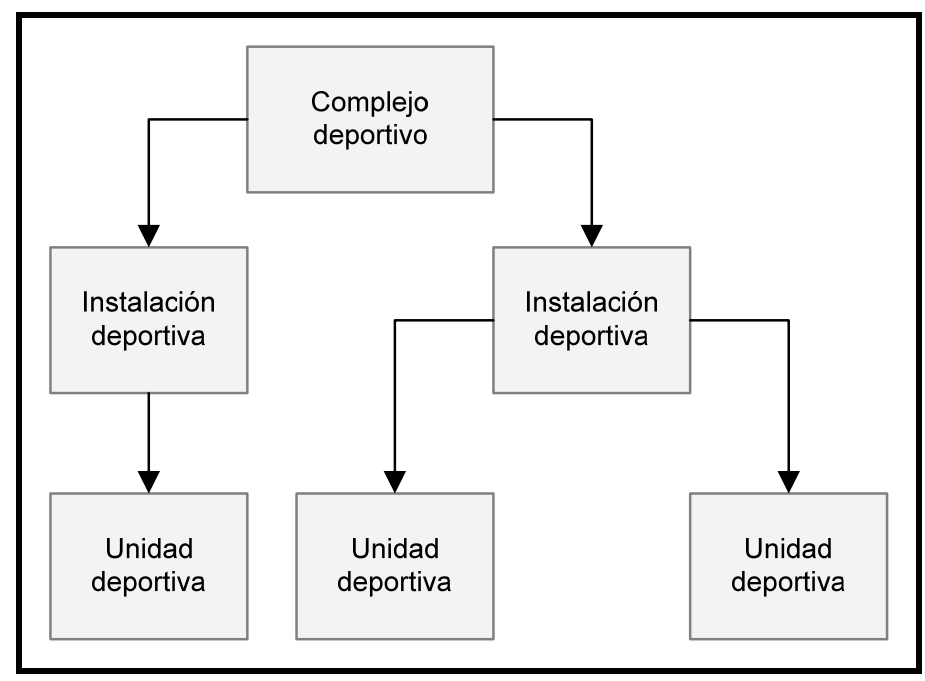

Fuente: Elaboración propia.

De igual manera se realizará un inventario de las actividades que quieran ser analizadas en el modelo, diferenciado la instalación se realizan y la unidad si se diera el caso. También se identificarán todos los costes que asume el SDM clasificándolos por naturaleza (de la forma que mejor se ajuste a las características de la organización), pudiendo así identificar el recurso vinculado a dichos costes y por lo tanto su finalidad, identificando de esta manera el lugar (centro o actividad) específico donde se han generado, para posteriormente agregarlos como costes directos o indirectos.

Fase 2 o fase de distribución. En esta fase se asignaran definitivamente todos los elementos de coste entre los centros de coste mediante un cuadro de reparto en el que aparezcan todos ellos. Se realizará de forma directa en los casos donde se identifico el centro exacto de origen y de forma indirecta entro los diferentes centros que hayan participado en su origen.

Para ello se utilizaran criterios de reparto basados en unidades físicas que representen de la forma más real posible la cuantificación del consumo que han 
realizado de los recursos que han originado los costes. De igual manera se realizaron los repartos pertinentes entre centros de costes mediante el mismo procedimiento, dadas las diferentes identificaciones que se les ha dado anteriormente y su diferente participación en el proceso productivo. En todo caso se asignarán los costes proporcionalmente al uso de la unidad de medida utilizada en el criterio de reparto. También se asignarán los costes vinculados a las actividades deportivas a las mismas, tanto de forma directa o indirecta si fuese necesario.

Fase 3 o fase de imputación. La última fase del modelo corresponderá a la determinación de los resultados finales. Para ello se imputarán los costes a las actividades deportivas por la utilización de las unidades deportivas, así como de otros centros de coste si se diera el caso. Entre los principales resultados se muestran el coste por hora de cada unidad deportiva. Además se pormenoriza en los cálculos de las actividades deportivas, llegando al coste unitario, ya sea por usuarios potenciales o por usuarios reales, teniendo en cuenta la posible desocupación (Tabla 2). De esta manera, se conocerán los costes totales de las unidades y de las actividades deportivas realizadas en las mismas, registrando toda la información mediante informes adecuadamente estructurados.

Tabla 2

Resultados principales previstos por la implantación del modelo

\begin{tabular}{|l|c|l|c|}
\hline Instalación Deportiva & Piscina Cubierta 1 & \multicolumn{1}{|c|}{ Actividad Deportiva } & Natación Adultos \\
\hline Unidad Deportiva & Vasos de Piscina Cubierta 1 & Unidad donde se realiza & Vasos de Piscina Cubierta 1 \\
\hline $\begin{array}{l}\text { Cose de bienes y } \\
\text { Servicios }\end{array}$ & - & Costes fijos & - \\
\hline Coste de personal & - & Costes variables & - \\
\hline Amortización & - & $\begin{array}{l}\text { Coste unitario por } \\
\text { usuarios reales }\end{array}$ & - \\
\hline Coste por hora & - & $\begin{array}{l}\text { Coste unitario por } \\
\text { usuarios potenciales }\end{array}$ & - \\
\hline
\end{tabular}

Fuente: Elaboración propia.

\subsection{Etapa 3. Realización del estudio de costes en los SDM}

En esta etapa se hace presente el trabajo del investigador en conjunto con los miembros de la organización. La labor del investigador consistió en ayudar al gestor en la coordinación del personal en la recopilación de información, además de asesorar de manera personal en la labor realizada por cada uno de los trabajadores, de manera que la información obtenida tenga las características necesarias para poder utilizarla en el modelo y conseguir los mejores resultados. La primera actuación a llevar a cabo fue informar a todo el personal de los principios y finalidades que se perseguían con esta propuesta, así como el tipo de información que debían reportar para su realización. Desde ese punto los traba- 
jadores debían recopilar y procesar la información de manera que se ajustara a las características demandadas.

Cabe destacar que a lo largo de esta etapa se tuvieron que realizar varios encuentros con grupos de trabajadores, dado que la idea sobre la filosofía y finalidad que adquirieron fue equivocada en algunos casos, dificultando el trabajo que realizaban para el estudio. Para lograrlo desarrollamos más ampliamente cada uno de los aspectos mencionados, haciendo referencia al proceso de cambio y las reformas de los servicios públicos municipales, en base a los principios del NPM y cómo afecta a áreas como los SDM. Para facilitar su entendimiento y la motivación hacía el trabajo en este estudio planteamos ejemplos de problemas que se están dando en este tipo de organizaciones, explicando la utilidad que se puede dar la información obtenida y conseguir la mejor solución a los mismos. De esta manera se consiguió obtener la información demandada y con la suficiente calidad, teniendo que realizar pocos cambios o puntualizaciones en la misma.

Tras conseguir la información básica para el modelo se procedió al cálculo y a la agregación del coste para llegar a obtener la información final. Durante este proceso se mantuvo contacto constante con todos los miembros de la organización para volver a concretar o pedir más indicaciones y poder adaptar la información al modelo planteado. Las adaptaciones realizadas en el borrador inicial fueron mínimas, destacando únicamente la propuesta de nuevos criterios de reparto en base a las características específicas de los SDM y en consenso con sus gestores. La facilidad otorgada por la inclusión de cetros anexos y centros auxiliares facilitó el tratamiento de las particularidades de cada uno de los SDM.

Por último se redactaron los informes atendiendo a las consideraciones realizadas por los gestores al inicio del proceso (etapa 1) y la finalidad del modelo. Cabe destacar que en dichos informes se facilitó una tabla donde se mostraba el desglose de todos los costes asignados a los centros y actividades, de manera que se podía observar la procedencia de cada uno de los costes que se le habían agregado y su división entre directos e indirectos, valorando la posible subjetividad asumida durante el proceso.

\subsection{Etapa 4. Valoración del estudio}

La percepción general sobre resultados del modelo propuesto por parte de los gestores de los SDM es satisfactoria, ya que la información responde a las utilidades previamente planteadas en la etapa 1. Además de las aportaciones específicas del estudio, se consigue avanzar hacia una nueva forma de pensar sobre la gestión en sus entidades, no solo por su parte sino por el resto de la organización. Son conscientes del potencial de sus servicios en el panorama municipal, así como de la influencia que tienen en su localidad. De igual ma- 
nera, son completamente partidarios de que se debe realizar cambios en la forma de gestionar sus servicios, adquiriendo nuevos principios, coincidiendo con la ideología del NPM. Las bases de esta iniciativa así como los resultados y los procedimientos llevados a cabo coinciden con este pensamiento, y facilitan el cambio y el avance hacía este nuevo tipo de gestión.

Entre las actuaciones llevadas a cabo durante el proceso, y que han originado esta respuesta, destaca la necesidad y el consejo por parte del investigador participante de aumentar la preocupación sobre el registro y procesamiento de información, no solo económica, sino cualquier información que refleje las actuaciones que está llevando la entidad, para posteriormente permitir la realización de estudios y propuestas como la que aquí se realiza y poder mantener un control de la entidad en todas sus dimensiones, actuando en consecuencia para mejorar cada una de ellas y la entidad en su conjunto.

Respecto a los resultados obtenidos a través del estudio de costes, en ambos casos se destaca como uso inmediato una propuesta de modificación o "adecuación de precios" a cada una de las actividades en función de los recursos que ha utilizado y su finalidad principal, e incluso realizar una reestructuración en sus actividades si se tuviera la oportunidad o se requiriera obligatoriamente en un momento dado. De nuevo se encuentran con el problema de que para su consecución, estas propuestas deberán ser evaluadas y consensuadas en diferentes órganos, tanto administrativos como políticos de sus correspondientes ayuntamientos, por lo que el inicio de su puesta en marcha no será conocido inicialmente, y lo más seguro es que no llegue a realizarse como se planteó.

Asimismo los gestores de los SDM comentaron que tenían una idea aproximada acerca de la rentabilidad y repercusión económica de sus instalaciones y actividades deportivas, pero que gracias a este tipo de iniciativa se ha podido ver reforzada esa idea, pudiendo cuantificar dicha información de forma estandarizada. Además permite mantener un control continuo mediante la periodización del estudio y la comparación.

Como se ha ido describiendo a lo largo de todo el análisis del proceso de implantación, han ido surgiendo una serie de dificultades y problemas que se han ido solucionando mediante diferentes estrategias, para poder finalmente asegurar la correcta implantación del modelo propuesto. De esta manera se define finalmente la metodología y el protocolo de nuestra aportación, la Factura en los Servicios Deportivos Municipales. La síntesis de todos los problemas encontrados, y sus soluciones vienen presentadas en la Tabla 3, por lo que se define un punto de referencia ante la realización de este tipo de estudios o de iniciativas similares en otros SDM. 
Tabla 3

Síntesis de los problemas encontrados y soluciones aportadas para su superación

\begin{tabular}{|c|c|}
\hline OBSTACULOS & SOLUCIONES \\
\hline \multicolumn{2}{|c|}{ ETAPA 1} \\
\hline $\begin{array}{l}\text { Falta de consenso inicial en la fijación de las } \\
\text { preferencias informativas, teniendo en cuenta como } \\
\text { debe ser tratada la desocupación de las instalaciones } \\
\text { deportivas }\end{array}$ & $\begin{array}{l}\text { Abarcar en ambos casos todas las posibilidades } \\
\text { abordadas, en este caso, cálculo en base a usuarios } \\
\text { reales y cálculo en base a usuarios potenciales o } \\
\text { plazas ofertadas }\end{array}$ \\
\hline $\begin{array}{l}\text { Dependencia de fuentes informativas externas al SDM } \\
\text { debido a la excesiva centralización informativa del } \\
\text { ayuntamiento }\end{array}$ & $\begin{array}{l}\text { Diseño de canales continuos de información con el } \\
\text { área competente, de manera que el SDM reciba } \\
\text { reporte detallado de los costes que ha originado }\end{array}$ \\
\hline $\begin{array}{l}\text { Dificultades en la fijación de plazos en la recopilación } \\
\text { de información, debido a la dependencia de otras } \\
\text { áreas y falta de experiencia en este tipo de iniciativas }\end{array}$ & $\begin{array}{l}\text { Establecer márgenes para el primer estudio, y } \\
\text { registrar el proceso por parte del SDM para tener } \\
\text { referencias en estudios posteriores y planificar el } \\
\text { estudio con gran exactitud }\end{array}$ \\
\hline \multicolumn{2}{|c|}{ ETAPA 2} \\
\hline $\begin{array}{l}\text { Dificultades para obtener un consenso sobre el } \\
\text { tratamiento de las instalaciones ante la duda de } \\
\text { dividirla por espacios }\end{array}$ & $\begin{array}{l}\text { Incluir un nuevo centro de coste principal con el } \\
\text { nombre de unidad deportiva, que será definida por los } \\
\text { gestores de los SDM en función de sus preferencias y } \\
\text { de las características de sus instalaciones }\end{array}$ \\
\hline $\begin{array}{l}\text { Detección de centros independientes o que no tienen } \\
\text { relación directa con el proceso de prestación de la } \\
\text { actividad deportiva }\end{array}$ & $\begin{array}{l}\text { Incluir dichos centros como centros anexos, de } \\
\text { manera que se le pueda dar el tratamiento que más } \\
\text { se adecua a sus características especificas }\end{array}$ \\
\hline \multicolumn{2}{|c|}{ ETAPA 3} \\
\hline $\begin{array}{l}\text { Confusión sobre la utilidad del cálculo de costes por } \\
\text { parte de los trabajadores }\end{array}$ & $\begin{array}{l}\text { Mantener reuniones de formación, en las que se } \\
\text { muestren ejemplos de la información adicional que } \\
\text { aporta y sus posibles usos }\end{array}$ \\
\hline $\begin{array}{l}\text { Dificultades en discriminar los costes que deberían } \\
\text { ser directos a instalaciones en un principio }\end{array}$ & $\begin{array}{l}\text { Utilizar inicialmente un criterio de reparto, e iniciar } \\
\text { acciones que mejoren el registro y procesamiento } \\
\text { continuo de información }\end{array}$ \\
\hline $\begin{array}{l}\text { Poca información de carácter no económico para ser } \\
\text { utilizada en los repartos de costes indirectos, debido } \\
\text { al poco control del funcionamiento de la organización }\end{array}$ & $\begin{array}{l}\text { Utilizar inicialmente los criterios de reparto que más } \\
\text { se aproximen. Realizar una propuesta de mediciones } \\
\text { continuas que faciliten el control y la realización de } \\
\text { estudios posteriores }\end{array}$ \\
\hline \multicolumn{2}{|c|}{ ETAPA 4} \\
\hline $\begin{array}{l}\text { Preocupación por la subjetividad obtenida en el coste } \\
\text { final, debido a los repartos realizados en el proceso }\end{array}$ & $\begin{array}{l}\text { Añadir tablas en los informes, donde aparezcan } \\
\text { desglosados todos los costes asignados a las } \\
\text { instalaciones deportivas, diferenciando su naturaleza } \\
\text { y su carácter de directo o indirecto }\end{array}$ \\
\hline
\end{tabular}

Fuente: Elaboración propia.

\section{DISCUSIÓN Y CONCLUSIONES}

En ambos casos se confirma la idea inicial de que los SDM son un área que encaja perfectamente con los principios del NPM, en cuanto que existe la posibilidad e indirectamente la obligación de ajustar unas tarifas en base a las características y finalidades de cada servicio, facilitando su sostenibilidad económica y viabilidad. Además facilita dirigir la atención al usuario en forma de consumidor o cliente, atendiendo a sus demandas y otorgándole el mejor servicio posible, con su valor correspondiente. La competitividad es por tanto una premisa obligada en la gestión actual de estos servicios, por lo que deben aprovechar al máximo sus posibilidades y ventajas, siempre teniendo en cuenta 
el objetivo de la promocionar y posibilitar la práctica de actividad física y deportes entre todos los sectores de la población.

Los gestores eran conscientes de la situación actual y de la necesidad de acogerse a esta nueva ideología. Coincidiendo con los resultados obtenidos por Yetano (2009) en la implantación del cuadro de mando en la administración local, los gestores consideran que el modelo propuesto es una herramienta útil y que coincide con la orientación de dichos principios, como son un mayor control de la entidad, la evaluación del rendimiento y una orientación al resultado. Además de ello, la información suministrada permite mejorar la planificación, tanto a corto como a largo plazo. La combinación de herramientas de control debería conseguir aumentar el potencial de cada una de ellas, y por lo tanto, mejorar el funcionamiento de los servicios.

Las diferencias en las actuaciones para satisfacer los intereses del sector político y de los clientes se hacen presentes en este estudio. Walker et al. (2011) destaca que una orientación de mercado en los servicios locales, en busca de la competitividad, aumenta la satisfacción del ciudadano, usuario de dichos servicios, pero tiene un efecto diferente entre el resto de partes interesadas. Al igual que ocurre con las organizaciones no lucrativas los SDM deben rendir cuentas a las diferentes partes interesadas, situadas a diferentes niveles de la organización (Saz Gil, 2008).

Se ha demostrado que para el correcto desarrollo de este tipo iniciativas, y del modelo propuesto en particular, se necesita de un gran trabajo por parte de la organización y los trabajadores. Coincidiendo con Arnaboldi y Lapsley (2003), no sólo se requiere un esfuerzo durante la implantación de un modelo de cálculo de costes, sino durante el trabajo diario en el constante registro y procesamiento de la información. Sin embargo, se debe resaltar que la preocupación por dicho trabajo facilitará la implantación posterior del modelo además de conseguir resultados de mayor calidad y facilitar la implantación de otro tipo de herramientas, como baterías de indicadores de rendimiento o un cuadro de mando integral.

Respecto a los problemas encontrados, cabe destacar la constante repetición de carencia de sistemas informativos de calidad, que aporten datos para implantar el modelo con al mayor exactitud. Este problema se repite en otras iniciativas semejante a la que aquí se expone, como por ejemplo durante la implantación de un sistema de indicadores de rendimiento realizado por Navarro Galera et al. (2008), destacando una excesiva centralización, la inexistencia de canales de información adecuados y falta de información sobre el consumo de recursos. En todos ellos existe coincidencia con nuestra investigación.

Es completamente necesario que tanto los gestores de los SDM como sus trabajadores comprendan la dirección de este tipo de propuestas, así como su vinculación con el NPM, ya que en adelante serán los encargados de implantar 
esta herramienta y otras nuevas, continuando el proceso de modernización y reforma (Ter Bogt y Van Helden, 2011).

Cabe destacar que en la literatura, tanto técnica como científica, es muy común la implantación de herramientas para la mejora de la gestión en base a las reformas correspondientes al NPM, basadas en el control y la evaluación del resultado, analizando su viabilidad. Sin embargo existe una carencia de investigaciones que evalúen las herramientas implantadas en sí, y si mejora realmente el rendimiento de la organización (Christensen et al., 2002). Estudios que evalúen SDM antes y después del proceso de reforma desde un punto de vista longitudinal, así como la comparación transversal entre SDM con diferentes estructuras mediante indicadores estandarizados podría ser un punto de partida idóneo para futuras investigaciones.

\section{REFERENCIAS BIBLIOGRÁFICAS}

ABERBACH, J. D. y CHRISTENSEN, T. (2005). "Citizens and consumers" en Public Management Review, 7(2), pp. 225-245.

AFONSO, A. y FERNANDES, S. (2006). "Measuring local government spending efficiency: Evidence for the Lisbon Region" en Regional Studies, 40(1), pp. 39-53.

ARNABOLDI, M. y LAPSLEY, I. (2003). "Activity based costing, modernity and the transformation of local government" en Public Management Review, 5(3), pp. 345-375.

AYUSO MOYA, A. y RIPOLL FELIU, V. M. (2005). "El estudio de casos como prototipo de la investigación en contabilidad de gestión desde una perspectiva cualitativa" en Revista Iberoamericana de Contabilidad de Gestión, 5, pp. 131-168.

BALAGUER-COLL, M. T.; PRIOR, D. y TORTOSA-AUSINA, E. (2010). "Decentralization and efficiency of local government" en The Annals of Regional Science, 45(3), pp. 571-601.

BARRETTA, A. y BUSCO, C. (2011). "Technologies of government in public sector's networks: In search of cooperation through management control innovations" en Management Accounting Research, 22(4), pp. 211-219.

BJØRNENAK, T. (2000). "Understanding cost differences in the public sector-a cost driver approach" en Management Accounting Research, 11(2), pp. 193211.

BOYNE, G.; MARTIN, S. y WALKER, R. (2004). "Explicit reforms, implicit theories and public service improvement" en Public Management Review, 6(2), pp. $189-210$. 
BUCH GÓMEZ, E. J. y CABALEIRO CASAL, R. (2011). "Hacia la determinación de la condición financiera de la administración pública local. Aplicación a los municipios de la comunidad autónoma de Galicia" en Academia, Revista Latinoamericana de Administración, 47, pp. 43-60.

BUDDING, G. T. (2004). "Accountability, environmental uncertainty and government performance: evidence from Dutch municipalities" en Management Accounting Research, 15(3), pp. 285-304.

CALDERA, J.; BAUJíN, P.; RIPOLL, V. y VEGA, V. (2007). "Evolución en la configuración de los sistemas de costeo basado en las actividades" en FACES, 14, pp. 13-28.

CARMO, L. P. F. y PADOVANI, F. (2012). "Advances and challenges for adoption of activity based costing (ABC) by public sector: a comparative study of Brazil, Colombia, Uruguay and United States" en Intemational Business Research, 5(2), pp. 150-158.

CARRASCO DÍAZ, C. (2011). Manual de Procedimiento para la implantación de un sistema de costes en la administración local. Madrid: FEMP.

CHRISTENSEN, T.; LAEGREID, P. y WISE, L. R. (2002). "Transforming administrative policy" en Public Administration, 80(1), pp. 157-178.

DAVIES, L. E. (2002). "Sport in the city: measuring economic significance at the local level" en European Sport Management Quarterly, 2(2), pp. 83-107.

DE LIU, Y.; TAYLOR, P. y SHIBLI, S. (2009). "Sport Equity: Benchmarking the performance of english public sport facilities" en European Sport Management Quarterly, 9(1), pp. 3-21.

FEMP (Federación Española de Municipios y Provincias). (2006). Guía para la implantación de un sistema de costes en la administración local. Madrid: FEMP.

GALLARDO, L. (2007). Censo Nacional de Instalaciones Deportivas de España-2005. Madrid: Consejo Superior de Deportes. Ministerio de Educación y Ciencia.

GALLARDO, L.; BURILLO, P.; GARCÍA-TASCÓN, M. y SALINERO, J. J. (2009). "The ranking of the regions with regard to their sports facilities to improve their planning in sport: the case of Spain" en Social Indicators Research, 94(2), pp. 297-317.

GARCÍA FERRANDO, M. y LLOPIS GOIG, R. (2011). Encuesta sobre los hábitos deportivos en España 2010. Ideal democrático y bienestar personal Madrid: Consejo Superior de Deportes. Centro de Investigaciones Sociológicas.

GIMÉNEZ, V. y PRIOR, D. (2007). "Long and Short-Term Cost Efficiency Frontier Evaluation: Evidence from Spanish Local Governments" en Fiscal Studies, 26(1), pp. 121-139.

HELMUTH, U. (2010). "Better performance with performance budgeting? Analyzing cases of success and failure in public administrations" en International Public Management Journal, 13(4), pp. 408-428. 
HOOD, C. (1991). "A public management for all seasons?" en Public Administration, 69(1), pp. 93-109.

HOOD, C. (1995). "The new public management in the 1980s: variations on a theme" en Accounting, Organizations and Society, 20(2/3), pp. 93-110.

IGAE (Intervención General de la Administración del Estado). (1994). Contabilidad analítica de las administraciones públicas. El proyecto CANOA. Madrid: IGAE.

IGAE (Intervención General de la Administración del Estado). (2004). Principios generales sobre contabilidad analítica en las administraciones públicas. Madrid: Ministerio de Economía y Hacienda.

JACKSON, A. y LAPSLEY, I. (2003). "The diffusion of accounting practices hte "managerial" public sector. International Journal of Public Sector Management, 16(5), pp. 359-372.

JÖNSSON, S. y LUKKA, K. (2007). "There and back again: doing interventionist research in management accounting". En C. S. Chapman, A. G. Hopwood y M. D. Shields (Eds.), Handbook of Management Accounting Research Volume 1 (pp. 373-397). Amsterdam: Elservier.

KLUVERS, R. y TIPPETT, J. (2011). "The views of councillors and managers on accountability in local government: An empirical study in Australia" en International Journal of Management, 28(2), pp. 519-527.

LAPSLEY, I., y PALLOT, J. (2000). "Accounting, management and organizational change: A comparative study of local government" en Management Accounting Research, 11(2), pp. 213-229.

MALMI, T. y GRANLUND, M. (2009). "In search of management accounting theory" en European Accounting Review, 8(3), pp. 597-620.

NAVARRO GALERA, A.; ORTIZ RODRÍGUEZ, D. y LÓPEZ HERNÁNDEZ, A. M. (2008). "Identifying barriers to the application of standardized performance indicators in local government" en Public Management Review, 10(2), pp. 241-262.

ORTIZ RODRÍGUEZ, D.; NAVARRO GALERA, A., y LÓPEZ HERNÁNDEZ, A. M. (2006). "Consensus among public manager as strategy for standarization of performance indicators" en International Public Management Journal, 9(4), pp. 371-398.

PADOVANI, E.; YETANO, A. y LEVY ORELLI, R. (2010). "Municipal performance measurement and management in practice: wich factors matter?" en Public Administration Quarterly, 34(4), pp. 591-635.

POLLANEN, R. M. (2011). "Relative performance benchmarking of local governments: case of Ontario municipalities" en International Journal of Business and Public Administration, 8(1), pp. 19-33.

RODRÍGUEZ BOLIVAR, M. P.; LÓPEZ HERNÁNDEZ, A. M. y ORTIZ RODRÍGUEZ, D. (2010). "Implementing the balanced scorecard in public sector agencies: An experience in municipal sport services" en Academia, Revista Latinoamericana de Administración, 45, pp. 116-139. 
SAZ GIL, M. I. (2008). "Introducción a la gestión Stakeholder en las organizaciones no lucrativas. Una aproximación a través del estudio de casos" en Estudios de Economía Aplicada, 26(1), pp. 191-214.

TER BOGT, H. J. y VAN HELDEN, G. J. (2011). "The role of consultant-researchers in the design and implementation process of a programme budget in a local government organization" en Management Accounting Research, 22(1), pp. 56-64.

VAN HELDEN, G. J.; AARDEMA, H.; TER BOGTC, H. J. y GROOT, T. L. C. M. (2010). "Knowledge creation for practice in public sector management accounting by consultants and academics: Preliminary findings and directions for future research" en Management Accounting Research, 21(2), 83-94.

VAN HELDEN, G. J. y NORTHCOTT, D. (2010). "Examining the practical relevance of public sector management accounting research" en Financial Accountability and Management, 6(2), pp. 213-240.

WALKER, R. M.; BREWER, G. A.; BOYNE, G. A. y AVELLANEDA, C. N. (2011). "Market orientation and public service performance: new public management gone mad?" en Public Administration Review, 71(5), pp. 707717.

WORTHINGTON, A. C. (2000). "Cost efficiency in Australian local government: a comparative analysis of mathematical programming and econometric approaches" en Financial Accountability and Management, 16(3), pp. 201-221

WORTHINGTON, A. C., y DOLLERY, B. E. (2002). "Incorporating contextual information in public sector efficiency analysis: a comparative study of NSW Local Government" en Applied Economics, 34, pp. 453-464.

YETANO, A. (2009). "Managing performance at local government level: the cases of the city of brisbane and the city of melbourne" en The Australian Journal of Public Administration, 68(2), pp. 167-181. 